\title{
THE VISUAL PERCEPTION OF SIZE AND DISTANCE
}

\author{
WALTER C. Gogel
}

Civil Aeromedical Research Institute, Federal Aviation Agency, Oklahoma City, Oklahoma

(Received 30 July 1962)

\begin{abstract}
The perception of absolute distance has been assumed to be important in the perception of the size of objects and the depth between them. A different hypothesis is proposed. It is asserted that perceived relative size and distance are the primary psychological phenomena with perceived absolute distance derived from the perceptual summing of perceived relative depths. In agreement with this point-of-view, it is stressed that relative rather than absolute retinal extents are the determiners of visually perceived extents. A principle called the "adjacency principle" is identified as perceptually organizing the relative retinal stimuli. This principle states that the apparent size or position of any object in the field of view is determined by whatever size or distance cues occur between it and adjacent objects. Some evidence for the adjacency principle is discussed and some consequences of the principle are considered.
\end{abstract}

Résumé-On admet généralement que la perception de la distance absolue est un facteur important de la perception de la dimension des objets et de la profondeur qui les separe. On propose une hypothèse différente, selon laquelle la dimension relative et la distance perçues sont les phénomènes psychologiques primaires, tandis que la perception de la distance absolue dérive d'une sommation des profondeurs relatives perçues. En accord avec ce point de vue, on insiste sur le fait que les écarts perçues visuellement sont déterminés plutôt par les écarts rétiniens relatifs que par les écarts absolus. On a identifié un principe, nommé "principe de contiguité", comme le facteur perceptuel qui organise les stimuli rétiniens relatifs. Ce principe établit que la dimension apparente ou la position d'un objet quelconque du champ visuel est déterminé par tout guide de taille ou de distance qui existe entre lui et les objets adjacents. On discute quelques faits qui sont en faveur du principe de contiguité et on étudie quelques conséquences de ce principe.

Zusammenfassung - Es wurde angenommen, dass die Wahrnehmung des absoluten Abstandes für die Wahrnehmung der Grösse und des Tiefenabstandes von Objekten bedeutsam sei. Eine neue Hypothese wird vorgeschlagen. Es wird behauptet, dass die wahrgenommene relative Grösse und der Abstand die primären psychologischen Erscheinungen sind, wobei der wahrgenommene absolute Abstand sich durch Summation der wahrgenommenen relativen Tiefenabstände in der Wahrnehmung herleitet. In Ubereinstimmung mit diesem Gesichtspunkt wird betont, dass vielmehr relative als absolute Abstände auf der Retina bestimmend für visuell wahrgenommene Ausdehnungen sind. Ein Prinzip, das "adjacency principle" (Nachbarschaftsprinzip) genannt wird, erweist sich als verantwortich für die wahrnehmungsgemässe Organisation der realtiven Reize auf der Netzhaut. Das Prinzip legt fest, dass die scheinbare Grösse oder Lage eines Objektes im Gesichtsfeld dadurch bestimmt ist, dass bei einer bestimmten Grösse oder einem bestimmten Abstand gewisse Marken zwischen ihm und dem benachbarten Objekt erscheinen. Eine Beweismöglichkeit für das Nachbarschaftsprinzip wird diskutiert und einige Folgerungen aus dem Prinzip werden betrachtet.

\section{INTRODUCTION}

EGOCENTRIC localization either explicitly or implicitly has been assumed to play a central part in the perception of size and distance. It is the purpose of this paper to question the validity of this point of view and to offer an alternative explanation of the factors important in perceived size and distance. This alternative explanation stresses that the relational characteristics of stimuli are the determiners of perception. It asserts that the perceptions

1 This paper is a modification of a paper presented by the author (while a member of the U.S. Army Medical Research Laboratory, Fort Knox, Kentucky) at the symposium on visual space perception (sponsored by the Armed Forces and the Committee on Vision of the National Research Council), Washington, D.C., 27 and 28 March 1961. 
of size and distance are determined by events unrelated to the observer's perception of his own position in the visual world. It further asserts that factors are operative to determine which of the many relational characteristics of visual stimuli are important for particular perceptions. One such factor, labeled the "adjacency principle", is identified and some consequences of the operation of this factor are discussed.

\section{EGOCENTRIC LOCALIZATION \\ AND THE VISUAL PERCEPTION OF SIZE AND DISTANCE}

\section{The Rôle of Egocentric Localization in the Perception of Relative Distance}

The possible rôle of egocentric localization in the perception of relative distance can be considered with the aid of Fig. 1. Figure 1 gives a notation for some physical sizes and distances associated with objects $e, f$ and $g$. A perceived distance can be enumerated for each of the physical distances $D_{e}, D_{f}$ and $D_{g}$. The perceived distances associated by the observer with the physical distances $D_{\ell}, D_{f}$ and $D_{g}$ will be labeled $D_{e}^{\prime}, D_{f}^{\prime}$ and $D_{g}^{\prime}$, respectively. Egocentric localization is involved in $D_{e}^{\prime}, D_{f}^{\prime}$ and $D_{g}^{\prime}$ since each is an observerperception of the distance of an obect from himself. Perceptions of this kind are termed perceptions of absolute distance or perceived absolute distances. The perceived depths

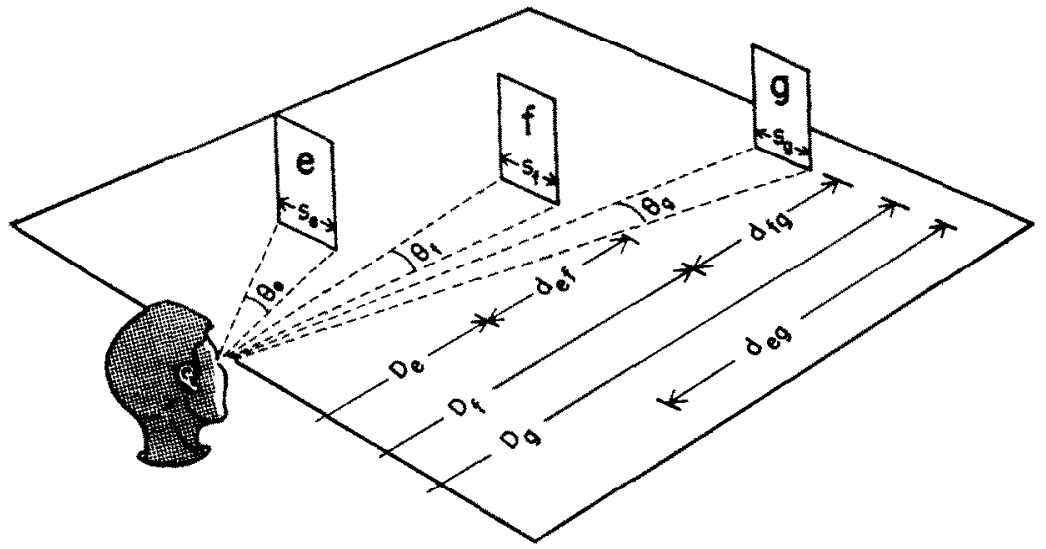

F1G. 1. A schematic diagram for considering perceptions of size and distance.

associated with the physical distances $d_{e f}, d_{f g}$ and $d_{e g}$ are perceptions of relative distance and will be labeled $d_{e f}^{\prime}, d_{f g}^{\prime}$ and $d_{e g}^{\prime}$. The explanation of $d_{e f}^{\prime}$ in terms of egocentric localization is that the observer arrives at $d^{r}$ ef by a process which is similar or equivalent to subtracting $D^{\prime}$ from $D_{f}^{\prime}$. Similarly, $d_{f g}^{\prime}$ would be explained as the consequence of a perceptual subtraction of $D_{f}^{\prime}$ from $D_{g}^{\prime}$, and $d^{\prime}{ }_{e g}$ as the consequence of a perceptual subtraction of $D_{e}^{\prime}$ from $D_{g}^{\prime}$. From this point of view, the perception of relative distance is a result of the difference between two perceptions of absolute distance. It follows, for example, that to the extent that $D_{e}^{\prime}$ and $D_{f}^{\prime}$ are ambiguous or impossible, $d_{e f}^{\prime}$ is ambiguous or impossible. According to this viewpoint, perceptions of absolute distance are the basic perceptual data, the immediate events, which determine the derived perceptions of relative distance.

An alternative hypothesis is possible. This hypothesis is that the perception of relative distance is the immediate event and that the perception of absolute distance, when it occurs, usually requires the perceptual summing of perceived relative distances. In this paper, some experimental evidence relevant to the two hypotheses will be examined. 
The hypothesis that perceived absolute distance determines perceived relative distance requires that cues be present which can directly determine perceived absolute distance. Visual cues of perceived absolute distance can be divided into the ocular cues such as convergence and accommodation and the so-called "empirical" cues such as the size cue associated with familiar objects. If perceived absolute distance is the determiner of perceived relative distance, it is reasonable to expect that direct and fairly precise visual cues of perceived absolute distance must always be available for the observer in situations in which perceived relative depth can occur. From this point of view it would also be expected that a change in perceived relative distance cannot occur without concomitant changes in perceived absolute distance. Both of these expectations require examination.

The convergence of the eyes as a cue to perceived absolute distance. The number of cues which can act directly in the sensing of the distance of an object from the observer are limited. The most important of these in situations involving only binocular cues are the convergence and accommodation of the eyes. The problem is whether accommodation and convergence per se are indicators of perceived absolute distance. Accommodation and convergence differences are not to be considered since these are possible direct indicators of relative not absolute depth.

The rôle of convergence and accommodation in the perception of distance has received considerable experimental attention (OSGOoD (1953), WOODWORTH (1938) and WOODWORTH and SCHLOSBERg (1954)). It is not always clear in this experimentation whether it is perceived relative or perceived absolute distance which is being studied, and whether accommodation and convergence differences or accommodation and convergence per se are the significant variables. However, a general conclusion which is possible from this work is that while convergence is more effective than accommodation, neither appears to be a very precise determiner of perceived absolute distance. In some recent experiments (GoGEL, $1961 \mathrm{a} ; 1961 \mathrm{~b} ; 1962 \mathrm{~b}$ ), perceived absolute distance was measured by providing the observer with a visual ruler consisting of a monocularly observed alley. This acted as a measuring stick against which the perceived distance of binocular configurations or of a single binocular object could be judged. In the experiments by Gogel, only a limited number of observers demonstrated any relation between convergence and perceived absolute distance. This was so whether a single binocular object or a configuration of binocular objects was used. Even for the observers who evidenced some relation between convergence and perceived absolute distance, the rate of change of perceived absolute distance with convergence was usually small and remained small whether a single binocular object or a configuration of binocular objects was used. Furthermore, for these successful observers, the convergence-perceived distance function differed, depending upon the position of the binocular objects in the configuration of binocular objects. Thus it appears that convergence is not a cue system which is effective with either sufficient frequency or precision to be a theoretical basis for the perception of relative depth resulting from a binocular disparity. This general inadequacy of convergence is pertinent to the Luneburg theory of binocular vision (Luneburg, 1947, 1948, 1950 and SHIPLEY, 1957). However, the fact that convergence does have some perceptual consequences with a few observers is also of significance. For example, the assumption that the addition of a constant amount of convergence will not affect the perceived depth in a binocular configuration (BLANK, 1957, 1959; HARDY et al., 1953 ) is neither true nor false. From the above studies by Gogel, it is true with most observers but not with all. 
Familiar size as a cue to perceived absolute distance. Another of the factors which has been considered to be a determiner of perceived absolute distance is the retinal size of a familiar object. If this cue operates with reliability and precision, then in a number of situations it offers the possibility that absolute distance perceptions are the necessary antecedents of perceived relative distance. The possible importance of this cue system is further enhanced by the circumstance that there are few (if any) other non-binocular cues, which might be direct determiners of perceived absolute distance, independent of perceptions of relative distance. Neither the relative size cue, nor its amplification into the perspective cue, nor the cue involving gradients of retinal size offer this possibility if size familiarity is not present.

According to the cue of familiar size, familiarity with an object, for example, a playing card, will result in a particular distance being associated with a particular retinal size of the playing card. This means that as a result of seeing playing cards at different distances, the observer will come to associate a particular retinal size of the card with a particular distance so that when only the retinal stimulus is present, the appropriate distance will be immediately perceived. If this hypothesis is correct, a familiar object in the absence of all other distance cues should be seen at a distance which varies inversely with its absolute retinal size (ItTelson, 1951, 1953 and Schlosberg, 1950).

But before considering familiar size as a cue to perceived absolute distance it is necessary to distinguish between relative and absolute retinal size. Consider the instance in which the eye is stimulated by a rectangle of light. For example, consider Object $e$ of Fig. 1. The retinal size, either angular $\left(\theta_{e}\right)$ or linear, of the width (or height) of this rectangle is an example of absolute retinal size. The ratio of the height to the width of the retinal image of the rectangle is an example of relative retinal size. Relative retinal size is involved when a successive or simultaneous comparison of retinal sizes is possible, while absolute retinal size might be measured as the number of millimetres or the number of receptor units subtended on the retina by the stimulus. The case of successive stimulation in the above definition of relative retinal size is designed to apply to the situation in which a stimulus, momentarily presented on the retina, is followed after a time interval by a stimulus of the same shape (or familiar characteristics) but of different retinal size. Suppose that, under these conditions, the observer perceives the retinally larger object as being closer than the retinally smaller object. What may be occurring in this case is a judgment of relative distance as a consequence of the successive presentation of the different retinal sizes. No judgments of absolute distance need be considered as having occurred. This example points out a caution which must be used in testing whether familiar size is a cue to perceived absolute distance. To be certain that absolute distance is being judged, the observer must not be permitted to compare retinal sizes either simultaneously or successively. An analysis of previous research on this problem in relation to these criteria is discussed elsewhere (GOGEL et al., 1957).

To meet the above requirements, different retinal sizes of a familiar object (a playing card) were presented to different observers and the perceived absolute distance of the card was measured kinesthetically without introducing a second visual object. The kinesthetic method of measurement consisted of throwing darts to the apparent distance of the playing card without knowledge of results. This method proved to have a precision which was more than adequate for the hypothesis being tested (GoGEL et al., 1957).

The results from this experiment were that the average distances thrown by the different groups of observers did not differ as a consequence of the different groups having been presented with different retinal sizes of the playing card. It follows that familiar size is not a 
cue to perceived absolute distance. This conclusion is also supported by a later study in which different retinal sizes of playing cards were used (GOGEL, 1960 a).

A review of the evidence relevant to familiar size as a cue to distance is given by EPSTEIN et al. (1961). In a number of previous studies, it had been concluded that familiar size can determine perceived absolute distance. In these studies the requirements listed above for measuring perceived absolute distance had not been met (GOGEL et al., 1957), and it is asserted that perceived relative not absolute distance was being studied. Also, a number of experiments have questioned the adequacy of familiar size as a cue to perceived distance (HochBer a and Hochber g, 1952, 1953; Hochberg and MCALISTER, 1955 and EPSTEIN, 1961). These experiments, however, involve the possible relation between familiar size and perceived relative, not perceived absolute, distance. If familiar size is not a cue to perceived relative distance, it is also not likely to be a cue to perceived absolute distance. The reverse is not necessarily the case, however. The demonstration that familiar size is not a cue to perceived absolute distance does not remove the possibility that it can affect the perception of relative depth.

The independence of perceived relative distance from perceived absolute distance. If perceived relative distance were to involve the subtraction of two perceived absolute distances, it is clear that changes in perceived absolute distance would be the necessary antecedents of changes in perceived relative distance. Direct evidence concerning this possibility is available. In the study of familiar size as a cue to perceived absolute distance (GoGEL et al., 1957), following the presentation of a particular retinal size of playing card, the other retinal sizes were presented successively to the same observers. Under these conditions, throwing differences occurred as a function of the changes in retinal size. The results indicate that perceived relative depth occurred from the successive presentation of different retinal sizes even though perceived absolute depth did not occur from the first presentations. Depth between the successive objects was perceived independently of any perception of the distance of any object from the observer. This experiment suggests that the perception of relative depth is immediate rather than a consequence of the difference between two perceptions of the distance of objects from the observer.

The physical depth interval (relative depth) associated with a constant binocular disparity varies approximately as the square of the distance of the objects from the observer. It is possible that, in the perception of a depth interval from binocular disparity cues, there is some perceptual process which parallels the relation between physical depth and binocular disparity. It might be expected that the perceived depth between two objects resulting from a binocular disparity would be some function of the perceived or estimated distance of the objects from the observer (FRY, 1950; OGLE, 1953, 1959 and von KRIES, 1925). Indeed, early experimental evidence was interpreted as supporting this point of view (HEINE, 1900). However, a more recent experimental test does not. In an experiment by GoGEL (1960 a), a ring of light was located a constant distance behind a pair of familiar objects (playing cards) with all of these objects binocularly observed in an otherwise dark room. The angular (retinal) size of the playing cards differed for two groups of observers. Measurements were made of both the perceived absolute distance to the playing cards and the perceived depth between the playing cards and ring. The two groups did not evidence any difference in the perceived absolute distance of the cards for the two angular sizes. The perceived depth between the cards and ring, however, was greater for the smaller size of the playing cards. It was concluded that the apparent depth between the ring and cards associated with a constant binocular disparity could be made to vary in spite of the fact that both the con- 
vergence and the perceived absolute distance of the cards were held constant. The importance of this experiment for the present discussion is that it indicates that a change in the perception of the absolute distance to a configuration of binocular objects is not a necessary condition for a change in the perception of the depth between them.

In another study, the perceptions resulting from a three-dimensional configuration were compared as a function of whether adcquate cues of absolute distance were present or absent (GoGEL, $1958 \mathrm{~b}$ ). The correlations between the results from the two types of situations again indicate that perceived absolute distance is not a determining condition for the three-dimensional perceptions resulting from a configuration of binocular objects. It appears from direct test that perceived absolute distance is not necessary to the perception of the relative depth between objects for either the binocular disparity cue or the cue of familiar size.

\section{The Rôle of Egocentric Localization in the Perception of Size}

The size-distance invariance hypothesis. The supposed dependence between perceived size and perceived absolute distance has found expression in what is termed the size-distance invariance hypothesis. This hypothesis states that the perceived absolute size $\left(S^{\prime}\right)$ of an object producing a constant retinal size $(\theta)$ is specified by the perceived absolute distance $\left(D^{\prime}\right)$. More specifically, the hypothesis is that

$$
S^{\prime}=K \theta D^{\prime}
$$

where $K$ and sometimes $\theta$ are constants (KILPATRICK and ITTELSON, 1953).

In equation $1, D^{\prime}$, a perception of absolute distance, is the perception by the observer that an object is at a particular distance from himself. In the present paper, the perception of absolute distance has been contrasted with the perception of relative distance, with the latter being the perceived distance between objects. Similarly, $S^{\prime}$, which is a perception of absolute size, can be contrasted with the perception of relative size, with the latter being the perceived ratio of the sizes of two objects. This distinction can be illustrated by Fig. 1. In Fig. $1, \theta_{e}, \theta_{f}$ and $\theta_{g}$ refer to the angular or retinal size of the widths of objects $e, f$ and $g$. The perceived absolute sizes associated with the widths of objects $e, f$ and $g$ are called $S_{f}^{\prime}$, $S_{f}^{\prime}$ and $S_{g}^{\prime}$ where $S_{e}, S_{f}$ and $S_{g}$ are the respective physical widths. When the sizes of two objects such as objects $e$ and $f$ are perceived relative to each other a perception of relative size or perceived relative size has occurred. This would be measured, for example, by adjusting the width of $S_{e}$ until it appeared equal to $S_{f}$. When the size of a visual object is judged by using some modality other than vision, however, a measure of perceived absolute size $S^{\prime}$ is approached, since a visual comparison between the visual object and the measuring device does not occur. For example, the kinesthetic modality could be used to measure perceived absolute size by having the observer adjust a non-visible distance between his two hands to apparently equal the width of the object seen in the visual field. This operational distinction between perceived absolute size and perceived relative size becomes clearer when the two types of measures are compared under the condition in which the perceived absolute size of all objects in the visual field is, for example, doubled. Under this condition, the relative size adjustment (the adjustment of the size of one visible object to apparently equal that of another visible object) would be unchanged whereas the kinesthetic adjustment would double. Thus, a kinesthetic adjustment method more nearly reflects an absolute judgment than does the method of adjusting one perceived visual size to that of another. 
The experimental evidence relevant to the size-distance invariance hypothesis has been reviewed by KilPATRICK and ITTELSON (1953) and by EPSTEIN et al. (1961). One method used to test the size-distance invariance hypothesis has been to correlate the results from a size constancy adjustment with the results from a distance fractionation judgment at a constant distance. Usually the correlations resulting from this process have not been significantly positive (CARLSON, 1960; GRUBER, 1954; JENKEN and HYMAN, 1959 and RUMP, 1961). When correlations between perceived size and perceived distance are determined as a function of distance, however, positive correlations though not necessarily a linear relation between perceived size and perceived distance have been found to occur (KURODA, 1961; Gogel, Wist and HARKer, 1962 and Rump, 1961). In the study by Gogel et al. (1962), measurements of perceived absolute size and of perceived absolute distance were determined with normal observation and also with decreased and increased interpupillary distances (base magnification). It was found that although perceived absolute size increased with increases in perceived absolute distance, the results do not support equation 1 . The ratio $S^{\prime} / D^{\prime}$ for a constant value of $\theta$ was found to vary significantly as a function of both physical distance and base magnification. A positive relation between $D^{\prime}$ and $S^{\prime}$ was indicated but the form of the relation was neither always linear nor necessarily constant for different viewing conditions. Perceived absolute distance and perceived absolute size may have a communality of determining events. But the experimental evidence does not support the hypothesis that the former necessarily determines the latter.

The previously discussed experiment which tested the hypothesis that the retinal size of a familiar object is a cue to perceived absolute distance is relevant here also (Gogel et al., 1957). The most likely reason for the failure of this hypothesis is that the retinal size of the familiar object was not a datum which could be used in the observer's perception. It is reasonable to expect that in the past history of the observers there were many opportunities to associate the absolute retinal sizes of playing cards with perceived absolute distances. The conclusion that such associations did not occur, therefore, suggests that the absolute size of the retinal image is not a datum of experience. It indicates that the absolute size of a retinal image has no perceptual consequences. But, the absolute retinal size $\theta$ is a possible variable in equation 1 ; i.e. according to equation $1, S^{\prime} / D^{\prime}$ is proportional to $\theta$. Thus the conclusion that the absolute retinal size of an image has no perceptual consequences is in opposition to the size-distance invariance hypothesis.

The convergence of the eyes as a determiner of perceived size. The judgment of the size of one visible object with respect to another visible object has been investigated in situations in which only binocular factors are present. In several of these studies, situations were used such that only convergence or convergence differences were available as possible distance indicators with binocular disparity absent (HEINEMANN et al., 1959; HeRMANS, 1954 and HOLWAY and BorING, 1941). The results obtained from these situations indicate that the ratio of the perceived sizes of the objects was not equal to the ratio of their retinal sizes. Some amount of size constancy was present, even when convergence was the only distance indicator available. It is possible to explain this size constancy in terms of two successive judgments of perceived absolute distance. From the size-distance invariance hypothesis, perceived absolute distance should determine the perceived absolute size associated with a retinal stimulus. Accordingly, as the perceived absolute distance increased the perceived absolute size associated with a constant retinal size would increase proportionately. If each perceived size were determined in this way, the comparison of sizes could also be explained by this process. But, from the previous discussion, perceived absolute distance usually will 
not occur as a function of convergence alone, and it is unlikely that absolute retinal size can determine perceived size. Therefore this explanation is doubtful. However, a direct test of this possibility has been made. The perceived absolute size of an object of constant angular size has been measured using a kinesthetic method, with convergence the only possible distance indicator (GOGEL, $1962 \mathrm{a} ; 1962 \mathrm{~b}$ ). The results indicate little or no change in perceived absolute size as a function of convergence. Thus the partial size constancy usually found with essentially only binocular cues present probably involves perceived relative size, resulting from perceived relative distance as a consequence of convergence changes, with perceived absolute distance not a significant factor.

\section{The Rôle of Egocentric Localization in the Perception of Shape}

From the discussion thus far, it appears that perceived size and perceived relative depth are not determined by perceived absolute distance even though, for a limited number of observers, these perceptions are not invariably independent of the cue of the convergence of the eyes. It might be expected that perceived three-dimensional shape, which can be regarded as a combination of a perceived frontoparallel size $\left(S^{\prime}\right)$ and a perceived relative depth $\left(d^{\prime}\right)$ would be similarly independent of perceived absolute distance and convergence. A direct test of this has been made in relation to the convergence of the eyes. In these experiments (GOGEL, $1958 \mathrm{a} ; 1958 \mathrm{c}$ ) the perceived three-dimensional shape of a binocular object was measured under conditions in which only binocular cues were present. It was found that the perceived shape varied somewhat as a function of the convergence value to the object. It is concluded from these results that convergence can have an effect upon perceived threedimensional shape.

\section{Discussion}

The evidence is that egocentric localization as it is reflected in perceived absolute distance is not the determiner of either perceived frontal size or perceived relative depth. Two of the cues which possibly could have been important in the direct perception of absolute distance are the convergence of the eyes and the retinal size of a familiar object. It is asserted that the absolute retinal size of a familiar object is not an adequate cue to perceived absolute distance. Convergence per se, in an imprecise manner, under certain circumstances, with some observers, can affect perceived absolute distance, perceived three-dimensional shape, and possibly perceived size. But, the last two effects, if and when they occur, are not determined by the first effect. It seems that under certain conditions, with certain people, convergence possibly can have some effect upon the perceptions of relative size and distance. But the effect is direct and does not involve perceived absolute distance as a necessary condition. This is further seen in experiments with a stereoscope in which convergence is sometimes found to affect perceived size and distance differently (WoodwORTH, 1938, pp. 674-676). It should also be kept in mind that apparently many people cannot use convergence effectively with respect to perceived absolute distance and perhaps perceived absolute size. The convergence of the eyes is not a very promising cue for asserting the direct importance of egocentric localization in the perception of size and distance.

The effect of apparent distance on the accommodation and convergence of the eyes has been investigated (Hofstetter, 1942, 1950, 1951; ItTELSON and AMES, 1950; MORGAN. $1944 \mathrm{a}, 1944 \mathrm{~b}$ and OHWAKI, 1955). It may be concluded that convergence and possibly accommodation can be affected by the apparent distance of the fixated object. It has been 
noted previously that a number of studies have been concerned with the possible relation between convergence and perceived size (GoGel, 1962 a, 1962 b; HEINEMANN et al., 1959; Hermans, 1954 and HolwaY and Boring, 1941). The possible relation between accommodation and perceived size has also been studied (Chalmers, 1952; HeinemanN et al., 1959; HolWAY and BorING, 1941 and OHWAKI, 1954). In some of these studies, it is not always clear whether the terms perceived size, perceived distance, accommodation, and convergence refer to absolute values of these events or to changes in their magnitudes. But to the extent that the accommodation-convergence complex affects the perception of relative size and distance and is in turn affected by perceived absolute distance, to this extent egocentric localization can have some effect upon perceived relative size and distance. However, the evidence is that a perception of absolute distance is not a necessary condition for the perception of either relative size or distance. At best it is a peripheral and indirect contributor to these perceptions and is not a basis for their theoretical explanation.

THE ADJACENCY FACTOR IN THE PERCEPTION OF SIZE AND DISTANCE

\section{The Relational Character of Stimuli}

In the previous discussion, the factors which were considered to be possible cues to absolute distance are non-relational. These were convergence (or accommodation) per se, rather than convergence (or accommodative) differences, and the absolute retinal size of a familiar object rather than the differences in retinal size between objects or between presentations. Thus cues for the direct perception of absolute distance are absolute in character rather than being relational. There is considerable experimentation which bears on the problem of the relational characteristics of stimuli as determiners of perception. This paper has also presented some evidence relevant to this issue. For example, it is suggested that the absolute size $\theta$ of a retinal image has no relation to either absolute or relative perceived size or distance. This is to be contrasted with relative retinal size; for example, the relative retinal size of the width to the height of object $e$ in Fig. 1. Perceptually, it is immediately clear even with object $e$ in isolation that object $e$ is higher than it is wide. The size of the width (or the height) of object $e$ on the retina is an absolute retinal size, the ratio of the width to the height is a relative retinal size. The latter, not the former, constitutes an adequate perceptual stimulus. It is a fundamental premise of this paper that perceived size and distance are determined by the relational characteristics of the stimuli. It will be the purpose of the remainder of this paper to discuss a factor which is important in organizing the relational characteristics into perceptions of size and distance. This factor will be called the "adjacency principle". The principle of adjacency determines how the relational characteristics of the stimuli are organized. It asserts that perceptions of relative size and distance are locally determined; e.g. they are determined by events which exist in the vicinity of the objects being judged and do not involve cues extending from the observer to the objects. This point of view when expressed in terms of retinal excitation means that there is a local autonomy on the retina with regard to perceptual effects. The perception of the sizes of two objects or of the depth between them is not determined by the entire retinal stimulation but rather by excitations usually occurring in the vicinity of the two retinal images of these objects.

\section{Directional Adjacency}

The adjacency principle can be divided into depth and directional adjacency. Experimental evidence is available with respect to each of these aspects. A study involving directional adjacency will be discussed with the aid of Fig. 2 . 
In Fig. 2 a, the broken lines $L_{L}$ and $L_{R}$ represent two lines of sight originating from the position of the observer with the observer's position being located below the figure. The line $A B$ represents a top view of the physical position of an object; for example, a window: while the line $C D$ represents a top view of the physical position of another object; for example, a long slender tube passing through the window so as to form the angle $\Phi$ with the plane of the window. For both object $A B$ and object $C D$ the left end of the object ( $A$ or $C$ ) is physically closer to the observer than the right end ( $B$ or $D$ ). All the objects in Fig. 2 are observed binocularly. Figure $2 \mathrm{~b}$ represents a top view of the apparent position of the same window and tube. As before, in distinction to physical events, perceived events will be indicated by the prime notation. In Fig. $2 \mathrm{~b}, A^{\prime} B^{\prime}$ represents a top view of the apparent orientation of the window while $C^{\prime} D^{\prime}$ represents a top view of the apparent orientation of

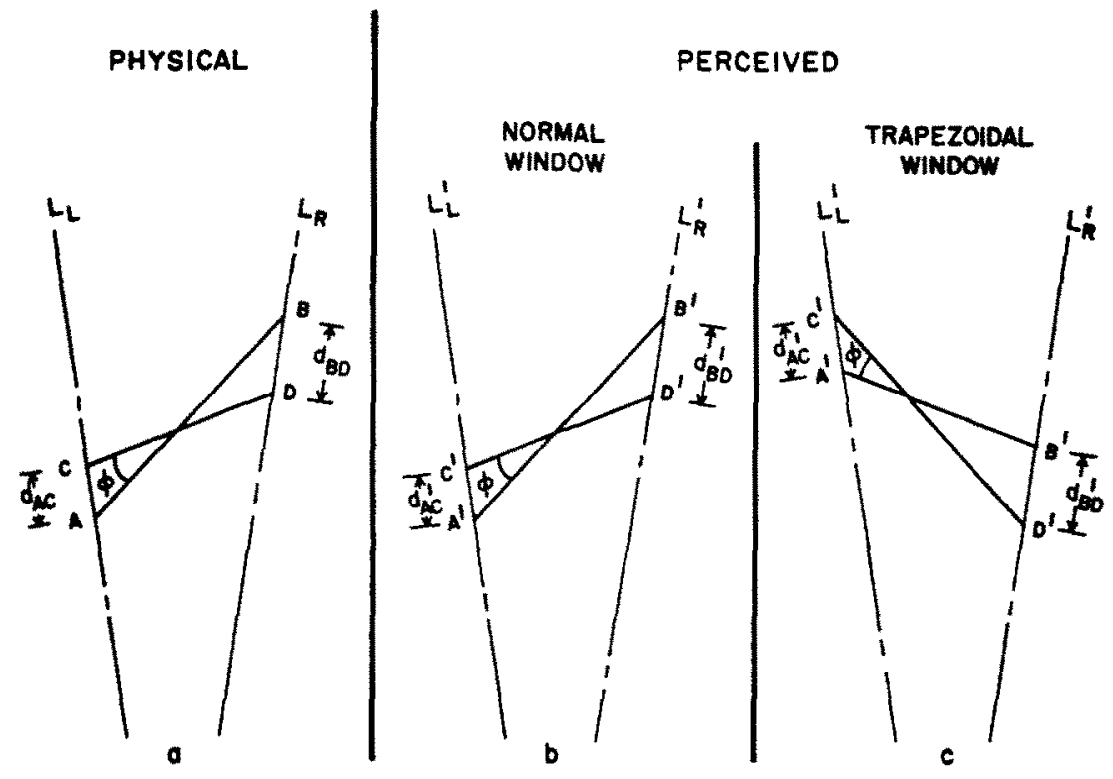

FIG. 2. The field effects of a visual distortion.

the tube. It will be noted by comparing Figs. $2 \mathrm{a}$ and $2 \mathrm{~b}$ that the perception of the orientation of the window and tube in Fig. $2 \mathrm{~b}$ is indicated as being veridical. It is meant by this that Fig. $2 \mathrm{~b}$ is drawn as though behavioral tests had revealed that the window and tube were perceived to be where they are. Suppose, however, that instead of a normally-shaped window an Ames trapezoidal window (AMEs, 1951) were used at $A B$ with the small end at $A$ and the large end at $B$ in Fig. 2 a. In this case, as is indicated in Fig. $2 \mathrm{c}$, the small end $A$ would appear to be behind the large end $B$ even though the reverse was physically true. What will be the apparent orientation of the tube $C D$ ? Experimentation on this type of problem has specified that the apparent orientation of $C D$ will be as shown in Fig. $2 \mathrm{c}$ with $d^{\prime}{ }_{\mathrm{AC}}$ and $d^{\prime}{ }_{\mathrm{BX}}$ having the same magnitude in Fig. $2 \mathrm{c}$ as in Fig. $2 \mathrm{~b}$ (GoGEL, $1956 \mathrm{~b}$ ). It will be noted in Fig. 2 that points $A$ and $C$ are always on the common line-of-sight $L_{L}$ while $B$ and $D$ are always on another common line-of-sight $\mathrm{L}_{\mathrm{R}}$. It can be generalized that the perceived depth resulting from the binocular disparity between points (or objects) along common lines-of-sight is unaffected by perceptual distortions occurring elsewhere. For example, point $C^{\prime}$ in Fig. $2 \mathrm{c}$ remains correctly perceived with respect to point $A^{\prime}$ but not 
with respect to points $B^{\prime}$ or $D^{\prime}$. It is as though the perceived position of point $C$ with respect to any other point is determined by its unchanged relation in perceived depth with respect to point $A$. The perceived depth position of $C$ is determined only by its line-of-sight binocular disparity with respect to $A$. More formally stated, the binocular disparity between any point (such as points $C$ or $D$ ) and that other point (such as points $A$ or $B$ ) which has the least separation in visual direction from the first point $(C$ or $D)$ will be the binocular disparity which is most effective in determining the perceived position of the first point $(C$ or $D)$ with respect to any other points in the field of view (GoGEL, $1956 \mathrm{~b}, \mathrm{p}$. 1). This principle applies to both Figs. $2 \mathrm{~b}$ and $2 \mathrm{c}$. However, only in the case of Fig. $2 \mathrm{c}$, i.e. only in the case of a perceptual distortion, does the problem as to which of the binocular disparities are perceptually most effective become obvious. It is concluded that the effectiveness of a binocular disparity in determining a perception of apparent position is inversely related to the relative directional separation between the points (or objects). As a limiting case, when the two objects (or points) are along an almost common line-of-sight the binocular disparity between these two points is almost completely dominant in determining their apparent position with respect to other points (or objects).

If this principle is applied in detail to Fig. 2, it will follow that if $A^{\prime} B^{\prime}$ in Fig. $2 \mathrm{c}$ is a straight line, $C^{\prime} D^{\prime}$ must have some slight curvature. Also, using the same principle, $\Phi^{\prime}$ in Fig. $2 \mathrm{c}$ will not equal $\Phi^{\prime}$ in Fig. 2 b. In general, Fig. 2 can be considered as illustrating a situation in which the perceived orientation of a vertical plane, defined in the top view drawing of Fig. $2 \mathrm{c}$ by $A^{\prime} B^{\prime}$, differs from its physical orientation ( $A B$ of Fig. $2 \mathrm{a}$ ). As a consequence of this perceptual distortion, all other planes in its vicinity (such as $C D$ ), which otherwise would have been correctly perceived, will also be distorted in perceptual orientation, with this distortion occurring in a systematic manner in agreement with the factor of directional separation discussed above.

The importance of directional separation, or rather of directional adjacency, in the perception of relative depth has been clearly demonstrated for the binocular disparity cue (GoGEL, 1954; 1956 b). It will be assumed pending further tests that this holds for all cue systems and that it can be generalized to the following statement: the relation between the perceived and the physical position of objects along a common line-of-sight is unaffected by distortions in perceived depth occurring elsewhere. A corollary is that the apparent position of an object in a configuration of objects is determined only by its line-of-sight relations. A more general statement is that the effectiveness of a cue system in determining the apparent relations (perceived size or distance) between objects (or between parts or points of objects) is inversely related to the magnitude of the relative directional separation between the objects (or between parts or points of objects). This is a statement of a principle of directional adjacency.

\section{Depth Adjacency}

In addition to the evidence for directional adjacency as an organizing factor in determining apparent position there is evidence for a factor of depth adjacency. The evidence comes from a series of experiments which have been concerned with the problem of specifying the factors which permit a binocular disparity to be interpreted as a depth extent. It was hypothesized in these experiments that the perceived size $\left(S^{\prime}\right)$ of objects in a frontoparallel plane (perceived frontal extents) in the vicinity of a binocular disparity determines the perceived depth associated with the binocular disparity (GoGEL, $1958 \mathrm{a} ; 1958 \mathrm{~b} ; 1958 \mathrm{c}$; $1959: 1960 \mathrm{a} ; 1960 \mathrm{~b}$ and $1960 \mathrm{c})$. It is as though the observer perceives the depth resulting 
from the binocular disparity to be a multiple of the width or height of an adjacent frontoparallel extent. More precisely, it was hypothesized (GOGEL, $1960 \mathrm{~b}^{2}{ }^{2}$ that the small perceived depth $\Delta d^{\prime}$ resulting from a small amount of binocular disparity $\Delta \alpha$ is

$$
\Delta d^{\prime}=\frac{S^{\prime}}{\theta} \frac{\Delta \alpha}{C}
$$

where $S^{\prime}$ is the perceived size and $\theta$ the angular (retinal) size of a frontally presented object (an object presented in a frontoparallel plane) at the same distance as the objects producing the binocular disparity, and $C$ is an individual constant. Equation 2 can be illustrated with the aid of Fig. 3 which is a schematic perspective diagram of an observer's viewing position

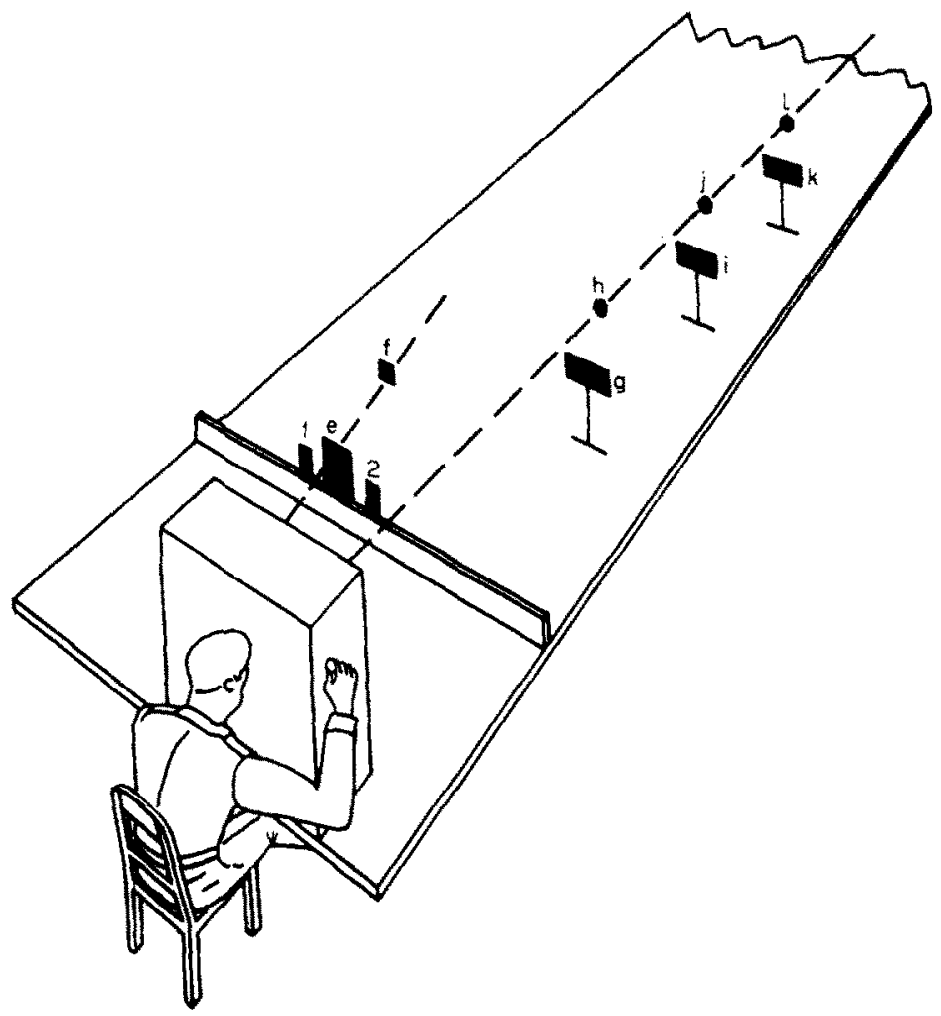

Fic. 3. Apparatus for a study of the interrelation of perceived size and distance. Reproduced with the permission of the Journal Press. It has appeared previously as FIG. 2 in GOGEL (1960 c).

and objects in a field of view. Consider the rectangle labeled $g$ and the small disc labeled $h$ located a very short distance behind $g$. As in Fig. 1, the angular size of the width of $g$ is $\theta_{g}$. The perceived depth between objects $g$ and $h$ in Fig. 3 is $\Delta d_{g h}^{\prime}$. The perceived width of object $g$ would be labeled $S_{g}^{\prime}$. Equation 2 states that

$$
A d^{\prime}{ }_{g h} / S_{g}^{\prime}=A \alpha_{g h} / C \theta_{g}
$$

or, the ratio of the perceived depth $\left(\Delta d_{g h}^{\prime}\right)$ to the perceived size $S_{g}^{\prime}$ of the frontal extent $S_{q}$ is equal to the ratio of the binocular disparity $\left(\Delta \alpha_{g h}\right)$ to the frontal angular size $\left(\theta_{g}\right)$ with $1 / C$

2 The original notation used in equation 2 has been changed to conform with the notation employed in this paper. In the original studies, $\Delta \alpha^{\prime}$ and $E$ were used instead of $\Delta d^{\prime}$ and $S^{\prime}$, respectively. 
being a constant of proportionality. In other words, equation 2 states that the ratio of perceived extents is proportional to the ratio of retinal extents.

It will be noted that in the discussion of equation 2 (Fig. 3) the $S^{\prime} / \theta$ value at object $g$, not at object $i$ or $k$, was used to determine the perceived depth between objects $g$ and $h$. Equation 2 specifies that a ratio of perceived extents is proportional to a ratio of retinal extents but only when the retinal extents arise from physical extents in the vicinity of each other. Apart from the constant $C$, the factor which determines $\Delta d^{\prime}{ }_{g h}$ is $S_{g_{i}}^{\prime} \theta_{g}$ (not $S_{i}^{\prime} \theta_{i}$ or $S_{k}^{\prime} / \theta_{k}$ ), the factor which determines $\Delta d^{\prime}{ }_{i j}$ is $S_{i}^{\prime} / \theta_{i}$ (not $S_{g}^{\prime} / \theta_{g}$ or $S_{k}^{\prime}{ }_{k} / \theta_{k}$ ), and the factor which determines $\Delta d^{\prime}{ }_{k l}$ is $S^{\prime}{ }_{k} / \theta_{k}$ (not $S_{g}^{\prime} / \theta_{g}$ or $S_{i}^{\prime} / \theta_{i}$ ). A depth adjacency effect is occurring. Not all perceived extents interact equally. In perceiving the depth associated with a binocular disparity, only perceptions involving frontal extents in the depth vicinity of the binocular disparity are important. It is asserted that this is not an act of conscious judgment but occurs as a consequence of the nature of the process by which a perceived depth results from a binocular disparity.

What is the evidence for the validity of equation 2 and thus for the validity of the depth adjacency factor? In an experiment discussed previously (GoGEL, 1960 a), the value of $\theta$ was changed with both the binocular disparity and $S^{\prime}$ essentially constant. It was found in agreement with equation 2 that as $\theta$ increased, the perceived depth between the binocular objects decreased. Equation 2 has also been applied to the comparison of depth intervals (GoGel, $1960 \mathrm{c}$ ). This also can be illustrated with Fig. 3. A constant binocular disparity between objects $e$ and $f$ was presented and was duplicated in apparent depth at $D_{g}, D_{i}$ and $D_{k}$, i.e. the observer adjusted $h, j$ and $l$ such that $d^{\prime}{ }_{g h}, d^{\prime}{ }_{i j}$, and $d^{\prime}{ }_{k l}$ each in turn equaled $d^{\prime}{ }_{e f}$. The $S^{\prime}$ values at $D_{g}, D_{i}$ and $D_{k}$ were measured by adjusting the lateral distance between objects 1 and 2 to duplicate the apparent width of the rectangles $g, i$, and $k$. It was found in agreement with equations derived from equation 2 , that the value of $S^{\prime} / \theta$ and the amount of binocular disparity required for the perceptually equal depth intervals were linearly related. The experiments concerned with three-dimensional, perceived shape (GOGEL, 1958 a; $1958 \mathrm{~b}$ and $1960 \mathrm{~b}$ ) support the same conclusion, i.e. equation 2 is in agreement with the obtained results.

It will be considered that the depth adjacency factor can be applied to cues in addition to that of binocular disparity. A statement of a principle of depth adjacency would then be as follows: the ratio of retinal extents arising from physical extents in the depth vicinity of each other determines the ratio of their perceived extents. This hypothesis asserts that frontal and depth extents in the same depth vicinity are perceptually interdependent such that for any cue to relative distance or perceived size, the specification of one also specifies the other.

\section{The Equidistance Tendency}

The importance of a relative directional factor in the organization of visual perceptions has also been demonstrated in the identification of what has been called the "equidistance tendency". If two or more objects are simultaneously presented in a visually reduced situation such that no cues are present to indicate the depth between the objects, they will appear to the observer to be at the same distance (equidistant). This is the equidistance tendency (GoGel, 1956 a). This tendency occurs in all visual situations but is masked by the presence of other distance cues. For example, if size cues exist between the objects, the effectiveness of the equidistance tendency will be reduced though probably not completely eliminated. It has been demonstrated that the equidistance tendency can modify the 
perceptual effectiveness of size cues (GOGEL and HARKER, 1955) and even under certain circumstances the cue of binocular disparity (GoGEL et al., 1954). The equidistance tendency can modify the perceived depth between either or both monocularly or binocularly observed objects.

Of importance for the present paper, the strength of the equidistance tendency has been found to be inversely related to the directional separation of objects. The closer the objccts are in visual direction, the stronger is the tendency for them to appear equidistant. This conclusion is meant to apply to both vertical and horizontal directional separations even though direct demonstrations have been made only with respect to horizontal separations. The equidistance tendency is another demonstration that directional adjacency is an important factor in the organization of the visual world. The effectiveness of the equidistance tendency in modifying perceived depth between objects varies inversely as the magnitude of the directional separation of the objects from each other.

\section{Discussion}

These studies in depth adjacency and in directional adjacency demonstrate that relationships between adjacent objects (or parts of objects) determine the perceived characteristics of these objects. The observer might wish to compare the relative position of two directionally non-adjacent objects independently of their relation to directionally adjacent objects, but he cannot. According to the principle of directional adjacency, the relations between directionally adjacent objects are dominant in determining apparent spatial position regardless of which other relations the observer attempts to use. Or, the observer might wish to judge one depth interval directly with another, disregarding the perceived size (per unit of retinal size) of nearby objects. But again he cannot do this. According to the principle of depth adjacency, the value of $S^{\prime} \mid \theta$ at a particular distance determines the perceived extent associated with a binocular disparity at that distance, regardless of the intent of the observer. Considering these two types of adjacency, and extending the adjacency concept to the perception of object size, it can be stated that the perceived depth intervals between objects or the perceived sizes of objects are determined by relations with respect to adjacent objects. Thus, what might be called the adjacency principle in three-dimensional perception can be stated as follows: the apparent position or apparent size of any object in a configuration of objects is determined by whatever cues or factors occur between the object and perceptually adjacent objects.

The adjacency principle emphasizes the interaction of local events and their independence from egocentric localization or other more remote events. In this process, use is made of some of our most precise visual abilities. These are the abilities to determine when objects are at the same or at a different distance (and direction) from each other. Some of these are such cues as zero amount of binocular disparity, the size cue involving equal retinal extents, the cue of overlay, etc. Thus precise cues are available for establishing perceptual adjacency and consequently for determining the interaction of locally perceived extents. Locally perceived extents then summate to produce larger perceptual intervals. These larger perceptual intervals are therefore derived extents and should tend to decrease in precision with an increase in the size of the interval.

Directional adjacency has been used successfully to predict the perceived path of movement in visually distorted fields. The perceived path of a moving object can be very different depending upon the arrangement of the object with respect to the adjacency factor (GoGEL, $1956 \mathrm{~b}$ ). Observers can be made to see an object as moving through a limited arc or as 
traversing an entire circle, depending upon this factor. Also, it must be emphasized that the effect of adjacency on perception does not occur by means of a conscious judgment. In situations as complex as those which have been used, e.g. in a situation in which objects or parts of objects are moving in different directions, it is clearly impossible for even a highly sophisticated observer to deduce rather than perceive a path of movement. It is asserted that adjacency is a principle which organizes perception quite independently of the thought process or the set of the observer.

It is also possible that the adjacency principle can be extended to the temporal as well as the spatial dimension. In the experiment involving the throwing of darts to the perceived absolute distance of playing cards (GoGEL et al., 1957), it was found that the temporal order in which the different retinal sizes were presented affected their perceived positions. This suggests that the order of presentation is perceptually significant. Consider the case in which the observer is presented with three different retinal sizes $A, B$ and $C$ with $A$ presented first and $\mathrm{C}$ last. According to the adjacency principle, the position (or size) of stimulus $\mathrm{A}$ with respect to stimulus $C$ is determined by whatever cues or factors are operative between stimuli $A$ and $B$ and between stimuli B and $C$. The perception of the distance (or size) of A with respect to $C$ must occur via the intermediary perceptions of $A$ with respect to $B$ and $B$ with respect to $C$. The adjacency principle asserts that the first stimulus (A) cannot be judged directly with respect to the last stimulus (C) but must occur by means of the perception of each of these stimuli with respect to the intermediate stimulus (B).

\section{IMPLICATIONS}

\section{The Veridicality of Size and Distance Cues}

The use of the concept of egocentric localization as a primary rather than a derived quality of spatial perception is congenial to the hypothesis that spatial perception is veridical. Suppose, for example, that the convergence or accommodation of the eyes were potentially adequate cues to perceived absolute distance. Through experience, the actualization of this potential could be adjusted so that perceived absolute distance and subsequently all perceived distances were veridical. Also, as discussed previously, if convergence produced veridical perceptions of absolute distance, this would at least offer the possibility that the perceived depth associated with a binocular disparity would also be veridical. A parallel discussion would apply with respect to the absolute distance cue of familiar size as a determiner of the perceived depth between familiar objects. But the concept of egocentric localization as a primary datum of perception has been rejected in this study. How veridical then are cues to perceived size and distance? If egocentric localization is a consequence rather than a determiner of perceived space, is perceived space veridical over a range of stimulus conditions? These questions will be considered with respect to the binocular disparity and the size cue to relative depth.

The binocular disparity cue to relative depth. There is considerable evidence that the perceived depth resulting from binocular disparity is not proportional to physical depth. As an example of this, consider the results from the study conducted with the apparatus illustrated in Fig. 3. As discussed previously, the observer adjusted object $h$ behind $g, j$ behind $i$, and $l$ behind $k$ until each of the depth intervals appeared equal to the perceived depth between objects $e$ and $f$. Only the binocular disparity cue was present between the pairs of objects to determine the perceived depth. From the results of this study, it was evident that perfect depth constancy was not present. The magnitude of the physical depth interval required in order to produce the constant perceived depth interval increased linearly with the distance 
of the depth interval from the observer (GoGEL, $1960 \mathrm{c}$ ). Perceived depth was not proportional to physical depth, and therefore the binocular disparity cue does not necessarily produce veridical depth perceptions.

The size cue to relative depth. From a study discussed in a previous portion of this paper it has been concluded that the absolute size of a retinal image has no perceptual consequences. Additional evidence to support this point of view is provided by a study by WALLACH and MCKENNA (1960) in which it was found that the perceived absolute size of a stimulus under conditions in which the visual cues were reduced was unrelated to perceived size as measured in more usual environments. Applying the conclusion concerning the perceptual inadequacy of the absolute size of a retinal image to the problem of perceived size, it is expected that only the ratios of retinal sizes (not their absolute sizes) are of importance in determining the perceived depth resulting from the cue of relative size. In agreement with this, Rock and EBENHOLTZ (1959) found that the perceived sizes of objects were dependent upon the size of surrounding objects (GIBSON, 1959, pp. 478-479). The conclusion concerning the importance of ratios of retinal size may be combined with the adjacency principle and applied to the perception of size. It follows that perceived size like the perceived depth resulting from a binocular disparity is determined by ratios of retinal sizes formed between adjacent objects. The application of this conclusion is illustrated with the aid of Fig. 1. Suppose in Fig. 1 that the size cue is the only cue present to determine the perceived depth associated with $\mathrm{d}_{e f}$ and $d_{f g}$. The retinal size of objects $e, f$, and $g$ is $\theta_{\ell}, \theta_{f}$ and $\theta_{g}$ respectively. From the requirement that relative retinal extents are the determiners of perception, ratios of $0 s$ rather than $\theta s$ per se are the significant stimuli. From the requirement that only adjacent stimuli are perceptually compared, the ratios of retinal sizes which are significant are those between physically adjacent objects. It follows that

$$
d_{e f}^{\prime}=d_{f g}^{\prime} \text { only when } \frac{\theta_{e}}{\theta_{f}}=\frac{\theta_{f}}{\theta_{g}}
$$

It is clear that equation 3 predicts that the perceived depth from size cues will not be veridical throughout the visual field. For example, for objects of equal physical size, if $D_{e}=10$ and $D_{f}=20 \mathrm{ft}, D_{g}$ would have to be $40 \mathrm{ft}$ in order to produce successively equal ratios of retinal size. In this case, $d_{f g}=2 d_{e f}$ when $d_{f g}^{\prime}=d^{\prime}$ ef. According to equation 3 , the perceived depth from size cues is not necessarily proportional to physical depth.

Figure 1 can also be used to illustrate the application of the adjacency principle to the perception of the large depth interval $d_{e g}$. It is asserted from the adjacency principle that the perceived extent $d^{\prime}$ eg would be determined by the two smaller perceived extents $d^{\prime}$ ef and $d_{f g}^{\prime}$ such that $d_{e g}^{\prime}=d^{\prime}{ }_{e f}+d_{f g}^{\prime}$. In fact, for a continuously filled visual field (and perhaps for any visual field) the perceived distance $d^{\prime}$ 'g would be determined by the perceptual summation of a large number of smaller intervals. However, consider only the case in which the perception of object $e$ with respect to object $g$ is a consequence of the two intermediate perceptions. This means that the perception of the depth position of object $e$ with respect to object $g$ is determined by whatever depth cues are available between objects $e$ and $f$ and between objects $f$ and $g$. Direct depth cues between objects $e$ and $g$ are trivial in specifying the perceived depth between these two objects.

\section{Psychophysical Equations of Perceived Space}

The previous discussion provides evidence for the importance of adjacency in the perception of space. It also sets some general requirements which psychophysical equations 
concerned with perceived space must meet. From the results of the experiment involving familiar objects and other considerations, it is concluded that the relational, not the absolute extents of retinal stimuli are determiners of perceived space. It follows from this that psychophysical equations involving perceived extents should contain terms for retinal extents, but only when these are in the form of ratios. ${ }^{3}$ It is also clear that these ratios should be between physically adjacent stimuli. In the previous discussion it has been assumed that physical (or perhaps retinal) adjacency has been the important adjacency factor. It is also possible that instead of either of these, it is perceptual adjacency which is the important quality. The resolution of this problem will require the investigation of situations in which each of these three forms of adjacency differ. However, for the purposes of the present discussion, it is sufficient to note that the adjacency principle requires that large perceived depth intervals are the summed effect of a series of small perceived depth intervals. From this point of view, the perception of large spacial extents is basically a process of perceptual summation. Specifically rejected is the notion that the perception of small extents is the result of a process of perceptual subtraction.

It has been found that equations derived from equation 2 were able to predict the median depth adjustments for the experiment discussed with the aid of Fig. 3 (GoGEL, $1960 \mathrm{c}$ ). The psychophysical equations predicted and the experimental results verified a linear contraction of perceived depth with respect to physical depth. This general discrepancy between the perceived and physical world has been noted in other experiments (GILINSKY, 1951). Clearly, not all systems of depth cues invariably produce veridical perceptions. This suggests that experience is not a determiner of the form of all psychophysical equations of perceived depth. It might be expected that experience can affect observer constants and scalar factors associated with psychophysical equations, e.g. factors like $C$ and $S^{\prime}$ in equation 2. But experience is not expected to affect the form of these psychophysical equations. Indeed, there seems to be no reason from the viewpoint of the effect of experience why the form of equation 2, for example, should be as it is. Experience would be expected to determine the form of a psychophysical equation by selectively reinforcing certain perceptions and not others. Those perceptions would be reinforced which tended to be in agreement with reality. Thus if the form of the psychophysical equation were determined by experience it would be expected that the psychophysical equation would predict veridical perceptions. Since this is not the case, it is suggested that the form of the psychophysical equation, as distinct from observer constants and scalar factors, is not the product of experience.

There is one other aspect regarding psychophysical equations of perceived space which might be mentioned. It is customary to enumerate the cues of space perception as those of binocular disparity, relative size, brightness, interposition, etc. However, it is possible that a more parsimonious set of variables might be established if the common aspects of these cue systems were investigated. If the perceived depth resulting from the different cue systems had the same phenomenal characteristics this would offer the possibility of some communality between them. It has sometimes been thought that binocular disparity produces an impression of depth, which is qualitatively different from that of other cue systems. There is evidence against this. Binocular disparity intervals have been used to measure perceived intervals developed by other cues such as that of relative size (GOGEL and HARKER, 1955). Apparently this can be done without any feelings of inappropriateness. This suggests that

${ }^{3}$ It will be noted that equation 2 , which contains a ratio of retinal extents, i.e. $\Delta \alpha / \theta$, meets this criterion. 
the quality of the depth experience is independent of the cue system used. This encourages the search for factors common to the various cue systems so that, for example, cue systems can be added together to produce perceptually predictable results.

\section{SUMMARY}

The concept of egocentric localization has been a central assumption in attempts to understand our visual perceptions of the three-dimensional world. This assumption is rejected. It is asserted that relative retinal events are the only events which can be stimuli for perceptions. These retinal events are organized by a factor which is termed the adjacency principle. The adjacency principle states that the apparent size or position of any object in the field of view is determined by whatever size or distance cues occur between it and adjacent objects. This point of view emphasizes the importance of local perceptual events and their summation into larger perceived extents rather than the reverse. It is suggested that experience can affect the observer constants and scalar factors in a psychophysical equation but it probably does not affect the form of the equation.

The adjacency principle has been applied to a situation involving perceived movement as well as to a number of static situations. It seems that the predictive ability of the principle is good under both types of circumstances.

\section{REFERENCES}

Ames, A., Jr. (1951). Visual perception and the rotating trapezoidal window. Psychol. Monogr. 65, No. 7. BLANK, A. A. (1957). A geometry of vision. Brit. J. physiol. Optics (3), 14, 222-235.

Blank, A. A. (1959). The Luneburg theory of binocular space perception. In Psychology: A study of a science (Ed. Koch, S.), Vol. I, pp. 395-426, McGraw-Hill, New York.

CARLSON, V. R. (1960), Overestimation in size-constancy judgments. Amer. J. Psychol. 73, 199-213.

Chalmers, E. L. (1952), Monocular and binocular cues in the perception of size and distance. Amer. $I$. Psychol. 65, 415-423.

Epstein, W. (1961). The known-size-apparent-distance hypothesis. Amer. J. Psychol. 74, 333-346.

EPSTEIN, W., PARK, J. and CASEY, A. (1961). The current status of the size-distance hypothesis. Psychol. Bull. 58, 491-514.

FrY, G. A. (1950). Visual perception of space. Amer. J. Optom. 27, 531-553.

GibSON, J. J. (1959). Perception as a function of stimulation. In Psychology: A study of a science. (KoCH, S., Fditor), Vol. I, pp. 456-501. McGraw-Hill, New York.

Gilinsky, A. S. (1951). Perceived size and distance in visual space. Psychol. Rev, 58, 460-482.

GOGEL, W. C. (1954). Perception of the relative distance position of objects as a function of other objects in the field. J. exp. Psychol. 47, 335-342.

Govel, W. C. (1956 a). The tendency to see objects as equidistant and its inverse telation to latcral separation. Psychol. Monogr. 70, No. 4,1-17.

Gogel, W. C. (1956 b). Relative visual direction as a factor in relative distance perceptions. Psychol. Monogr. 70, No. 11, 1-19.

GOGEL, W. C. (1958 a). An observer constant in the perception of stereoscopic depth. USAMRL Report No. 316, 1-24, Fort Knox, Kentucky.

GOGEL, W. C. (1958 b). The perception of shape from binocular disparity cues. USAMRL Report No, 331, 1-27, Fort Knox, Kentucky.

GoGEL, W. C. (1958 c). The effect of convergence and angular size upon the computed value of a hypothetical observer constamt in binocular vision. USAMRL Report No. 372, 1-28. Fort Knox, Kentucky.

GOGEL, W. C. (1959). The perception of space with binocular disparity cues. USAMRL Report No. 379, 1-24, Fort Knox, Kentucky.

Gogel, W. C. (1960 a). Perceived frontal size as a determiner of perceived binocular depth. J. Psychol. 50, $119-131$.

Gogel, W. C. (1960 b). The perception of shape from binocular disparity cues. J. Psychol. 50, 179-192.

Gogel, W. C. $(1960 \mathrm{c})$. The perception of a depth interval with binocular disparity cues. $J$. Psychol. $\mathbf{5 0}$, 257-269.

Gogel, W. C. (1961 a). Convergence as a cue to absolute distance. J. Psychol. 52, 287-301.

GogtL, W. C. (1961 b). Convergence as a cue to the perceived distance of objects in a binocular configuration. J. Psychol. 52, 303-315. 
GoGEL, W. C. (1962 a). Convergence as a determiner of perceived absolute size. J. Psychol. 53, 91-104.

GogeL, W. C. (1962 b). The effect of convergence on perceived size and distance. J. Psychol. 53, 475-489.

GOGEL, W. C., BRUNE, R. L. and INABA, K. (1954). A modification of a stereopsis adjustment by the equidistance tendency. USAMRL Report No. 157, 1-11, Fort Knox, Kentucky.

Gogel, W. C. and HARKER, G. S. (1955). The effectiveness of size cues to relative distance as a function of lateral visual separation. J. exp. Psychol. 50, 309-315.

Gogel, W. C., HARTMAN, B. O. and HARKER, G. S. (1957). The retinal size of a familiar object as a determiner of apparent distance. Psychol. Monogr. 71, No. 13, 1-16.

Gogel, W. C., WIST, E. R. and HARKER, G. S. (1962). A test of the size-distance invariance hypothesis. USAMRL Report No. 545, 1-23, Fort Knox, Kentucky.

Gruber, H. E. (1954). The relation of perceived size to perceived distance. Amer. J. Psychol. 67, $411-426$.

Hardy, L. H., Rand, G., Rittler, M. C., Blank. A. A. and Boeder, P. (1953). The geometry of binocular space perception. Report from the Knapp Memorial Laboratories, Institute of Ophthalmology, Columbia University College of Physicians and Surgeons, New York.

HEINE, L. (1900). Uber "Orthoskopie" oder Uber die Abhängigkeit relativer Entfernungs-schätzungen von der Vorstellung absoluter Entfernung. Arch. v. Graefes Ophthal. 51 (3), 563-572.

HeINemanN, E. G., Tulving, E. and Nachmias, J. (1959). The effect of oculomotor adjustments on apparent size. Amer. J. Psychol. 72, 32-45.

HERMANS, T. G. (1954). The relationship of convergence and elevation changes to judgments of size. J.exp. Psychol. 48, 204-208.

Hochberg, C. B. and Hochberg, J. E. (1952). Familiar size and the perception of depth. J. Psychol. 34, $107-114$.

HochberG, C. B. and Hochberg, J. E. (1953). Familiar size and subception in perceived depth. $J$. Psychol. 36, 341-345.

Hochberg, J. E. and MCAlister, E. (1955). Relative size vs. familiar size in the perception of represented depth. Amer. J. Psychol. 68, 294296.

HofstetTeR, H. W. (1942). The proximal factor in accommodation and convergence. Amer. J.Optom. 19, $67-76$.

Hofstetter, H. W. (1950). Accommodation, convergence and their relation to apparent distance: A criticism. J. Psychol. 30, 393-394.

HOFSTETTER, H. W. (1951). The relationship of proximal convergence to fusional and accommodative convergence. Amer. J. Oplom. 28, 300-308.

Holway, A. H. and BoRING, E. G. (1941). Determinants of apparent visual size with distance variant. Amer. J. Psychol. 54, 21-37.

Ittelson, W. H. (1951). Size as a cue to distance: Static localization. Amer. J. Psychol. 64, 54-67.

ItTElson, W. H. (1953). A note on familiar size and the perception of depth. J. Psychol. 35, $235-240$.

ItTELSON, W. H. and AMES, A., Jr. (1950). Accommodation, convergence and their relation to apparent distance. J. Psychol. 30, 43-62.

JENKIN, N. and HYMAN, R. (1959). Attitude and distance-estimation as variables in size-matching. Amer. J. Psychol. 72, 68-76.

Kilpatrick, F. P. and Ittelson, W. H. (1953). The size-distance invariance hypothesis. Psychol. Rev. 60, $223-231$.

Kuroda, T. (1961). Experimental studies on size constancy. In Bulletin of the Faculty of Literature of Kyushu University, No. 7 (Akishige, Y., Editor), Fukuoka. Japan.

Luneburg, R. K. (1947). Mathematical analysis of binocular vision. Princeton University Press, Princeton, N.J.

Luneburg, R. K. (1948). Metric methods in binocular visual perception. In Studies and Essays: Courant Anniversary Volume, Interscience, New York.

LUNEBURG, R. K. (1950). The metric of binocular visual space, J. opt. Soc. Amer. 40, 627-642.

Morgan, M. W., Jr. (1944 a). Accommodation and its relation to convergence. Amer. J. Optom. 21, 183-195.

Morgan, M. W., Jr. (1944 b). The clinical aspects of accommodation and convergence. Amer. J. Optom. 21, 301-313.

OGLE, K. N. (1953). Precision and validity of stereoscopic depth perception from double images. J. opt. Soc. Amer, 43, 906-913.

OGLe, K. N. (1959). Theory of stereoscopic vision. In Psychology: A study of a science (KocH, S., Editor), Vol. I, pp. 362-394, McGraw-Hill, New York.

OHWAKI, S. (1954). On the role of accommodation in the size constancy phenomenon. Tohoku psychol. Fol., $14,17-31$.

OHWAKI, S. (1955). On the factors determining accommodation: Research on size constancy phenomenon. Tohoku psychol. Fol. 14, 147-158.

Osgood, C. E. (1953). Method and theory in experimental psychology. Oxford University Press, New York. 
Rock, I. and Ebenholtz, S. (1959). The relational determination of perceived size. Psychol. Rev. 66. $387-401$.

RUMP, E. E. (1961). The relationship between perceived size and perceived distance. Brit. J. Psychol. 52. 111-124.

SCHLOSBERG, H. (1950). A note on depth perception, size constancy, and related topics. Psychol. Rer. 57. $314-317$.

ShIPLEY, T. (1957). Convergence function in binocular space; I. A note on theory. J. opt. Soc. Amer. 47, $795-803$.

vON KRIES, J. (1925). Notes on perception of depth. In H. V. HeLmHoltz's Treatise on Physiological Optics, Vol. III (Southall, J. P. C., Editor), Banta, Menasha, Wisconsin.

WALLACH, H. and MCKENNA, V. V. (1960). On size-perception in the absence of cues for distance. Amer. J. Psychol. 73, 458-460.

Woodworth, R. S. (1938). Experimental Psychology. Henry Holt and Co., Inc., New York.

Woodworth, R. S. and Schlosberg, H. (1954). Experimental Psychology. Henry Holt and Co., Inc.. New York. 